\title{
Accidentes en la infancia: Su prevención, tarea prioritaria en este milenio
}

\author{
PATRICIO ROMERO P. ${ }^{1}$ \\ 1. Departamento de Pediatría y Cirugía Infantil Sur. Facultad de Medicina Universidad de Chile. Comité Traumatismos, \\ Envenenamientos y Violencias. Sociedad Chilena de Pediatría.
}

\begin{abstract}
Accidents in pediatrics: the millennium challenge

Accidents and violence represent in Chile and Latinoamerican countries one of the leading mortality causes. Epidemiologic features of these health problems are described, with emphasis in prevention strategies.

Key words: chilhood accidents, epidemiology, traumatisms, violence, strategies, intervention.

Rev Chil Pediatr 2007; 78 (Supl 1): 57-73

\section{RESUMEN}

Los traumatismos derivados de accidentes y violencias continúan representando en Chile y en muchos otros países latinoamericanos, la principal causa de muerte, entre los mayores de un año y hasta la edad adulta (46 años). Son importante causa de ingresos hospitalarios y consultas a los servicios de urgencia. Se describen las características epidemiológicas de accidentes y violencias, los accidentes y amenazas más frecuentes y los más notables que por su gravedad y trascendencia afectan al niño chileno. Se hace énfasis en la prevención de los más importantes y se enumeran las estrategias de intervención propuestas.

(Palabras clave: Accidentes infantiles, epidemiología, traumatismos, violencias, estrategias, intervención).

Rev Chil Pediatr 2007; 78 (Supl 1): 57-73
\end{abstract}

Los traumatismos derivados de accidentes y violencias continúan representando en Chile, y en muchos otros países latinoamericanos, la principal causa de muerte entre los niños mayores de un año, circunstancia que por lo demás se extiende hasta la edad adulta (46 años). Se estima que por cada muerte por trauma, se provocan 18 ingresos hospitalarios y alrededor de 200 consultas a los servicios de urgencia.

Aunque el total de niños lesionados en nuestro país ha disminuido globalmente, su relevancia como problema de salud pública persiste y sus consecuencias continúan siendo de la mayor trascendencia por su magnitud e impacto 
en cuanto a sufrimiento humano, a los notables costos sociales y económicos para la familia, el estado y las organizaciones de salud privadas, así como a las discapacidades físicas y psíquicas que afectarán a la víctima y su entorno.

La prevención de los accidentes es un tarea fundamentalmente educativa y formativa, en la que necesariamente se considerarán además, la utilización de recursos de ingeniería, diseño y tecnología, de seguridad ambiental, control y vigilancia, formulaciones legislativas y judiciales, que permitan proponer y obtener los cambios de conductas en las personas, anticipar e identificar las situaciones o acciones de riesgo, reconocer los ambientes peligrosos, procurando la protección de niños que por su edad o condición, no sean capaces de reconocer los riesgos para su salud e integridad.

En este contexto, los profesionales de la salud, así como las sociedades que los agrupan, adquieren un rol principal en la tarea preventiva, en cuanto a considerar como significativa, su opinión técnica y el consejo educativo y normativo que logren difundir.

En naciones desarrolladas se han aplicado con éxito programas de intervención que muestran una importante disminución de la mortalidad por esta causa. Esta se calcula en alrede- dor del 30\%, aplicando determinados modelos preventivos. Las estrategias y resultados de su aplicación se observan en la tabla 1.

Sin embargo, estimaciones en la población general de la contribución de cinco factores de riesgo en colisiones del tráfico en Estados Unidos (EUA) entre los años 1982 y 2001, demostró que disminuir el uso de alcohol entre los conductores y el aumento en la utilización del cinturón de seguridad por ocupantes, provocó una sustancial reducción en la mortalidad por choques vehiculares.

Desde una mirada más general, se reconoce la particular vulnerabilidad de los niños a los riesgos ya no sólo por traumas por accidentes y violencias, sino además por otras amenazas ambientales, y esta convicción ha impulsado la promoción y el desarrollo de iniciativas de carácter y estrategias más globales que permitan a los niños vivir en ambientes más seguros y saludables.

La Organización Mundial de la Salud (OMS) promueve desde hace algún tiempo la formación y organización de los Centros de Salud de Pediatría Ambiental cuyos propósitos son procurar la apropiada información, la investigación, el diagnóstico epidemiológico, las medidas de prevención primaria así como las estrategias

Tabla 1. Circunstancia, estrategia de prevención aplicada y estimación del porcentaje de vidas salvadas en EUA*

\begin{tabular}{llc}
\hline Circunstancia & Estrategia & $\begin{array}{c}\text { \% } \\
\text { Dismución }\end{array}$ \\
\hline Ocupantes de vehículos & Bolsas de aire, cinturones de seguridad & 40,0 \\
Peatones & Educación a la comunidad & 39,2 \\
Motociclistas & Cascos & 15,1 \\
Ciclistas & Cascos & 65,9 \\
Inmersión & Rejas en piscinas, prevención de accidentes en bañeras & 58,4 \\
& Control sobre el uso de alcohol en adolescentes & 63,2 \\
Quemaduras & Detectores de humo, cigarrillos auto extinguibles & 59,6 \\
Intoxicaciones & Envases seguros, control de muerte por CO & 42,1 \\
Caídas & Rejas en ventanas & 36,8 \\
Violencia por armas de fuego & Eliminar o poner en resguardo armas de fuego en el hogar & 31,1 \\
Total & & \\
\hline
\end{tabular}

* Estados Unidos de América 
de manejo, de los problemas medioambientales identificados y que afecten a las distintas regiones, entregando además educación, capacitación y apoyo técnico a los autoridades e instituciones involucradas.

En este mismo contexto dicha organización ha planteado, por ejemplo, a requerimiento de la Comunidad Europea, y en el marco de la Cuarta Conferencia Ministerial de Ambiente y Salud 2004 denominada "El futuro de nuestros niños", una serie de recomendaciones incluidas en un documento, denominado, Children's Environment and Health Action Plan for Europe (CEHAPE).

Este plan de acción destaca cuatro postulados de salud prioritarios y principales para estos países, y que en consideración a las informaciones disponibles de la realidad sanitaria en América del Sur y Caribe, pueden ser también aplicados, con los necesarios resguardos, a nuestras comunidades. Se plantearon entonces los siguientes propósitos principales:

- Proveer la disposición de agua limpia y segura, que permita prevenir y reducir, la morbimortalidad por trastornos gastrointestinales y otros efectos sobre la salud infantil.

- Asegurar la protección contra lesiones, evitando y disminuyendo las consecuencias de traumas y violencias sobre la salud de los niños, y promoviendo además una adecuada y segura actividad física, desarrollada en ambientes y condiciones apropiadas, libre de riesgos, y que aminore asimismo las consecuencias implícitas de una vida sedentaria.

- Prevenir y reducir la enfermedad respiratoria, atribuida a la contaminación intra y extra domiciliaria y asegurando el derecho de todos los niños de vivir en un ambiente no contaminado.

- Disminuir el riesgo de enfermedad y discapacidad derivados de la exposición a agentes químicos (metales pesados por ejemplo), agentes físicos, a trabajos y ambientes peligrosos, para niños y embarazadas.

Parece obvio que estos postulados no sólo son aplicables para poblaciones infantiles de regiones de mejor nivel socioeconómico, sino que con mayor urgencia y dramática necesidad, aunque con diferentes prioridades, también para las regiones del tercer mundo. Bastará reconocer las realidades epidemiológicas de países sudamericanos, afectados por graves problemas sanitarios y de salud ambiental, donde aún la diarrea, la desnutrición y las enfermedades respiratorias bajas, son principal causa de mortalidad infantil.

De gran utilidad será entonces promover proyectos que aseguren, por ejemplo, la adecuada disposición de agua potable y apropiada habilitación sanitaria, especialmente entre las poblaciones rurales y en aquellas de pobreza extrema, distribuidas extensamente en la región de América Latina y el Caribe.

Al analizar las principales causas de mortalidad en menores de 5 años en las poblaciones de las regiones mencionadas (figura 1), es razonable colegir que las estrategias de intervención en prevención primaria que se puedan organizar, poner en marcha y aplicar sobre las enfermedades respiratorias, lesiones por accidentes, enfermedades infecciosas y desnutrición, todas ellas esencialmente prevenibles, y que corresponden aproximadamente al $30 \%$ de todas las causas de muertes estimadas, provocará un impacto enorme en cuanto a la disminución de las cifras de morbi-mortalidad.

Para encarar integralmente el problema en la región, probablemente se requiera definir una adecuada clasificación de los distintos países que la conforman, ordenándolos de acuerdo a diferentes parámetros que los agrupen en relación por ejemplo a sus tasas de morbi-mortalidad infantil, y otros medidores e indicadores que identifiquen diferencias en lo económico, social, político y demográfico, de un determinado país o sub región, y permita reconocer los riesgos de cada población. Con estos antecedentes se podrán definir programas, estrategias e intervenciones que permitan disminuir el impacto sobre una determinada población o región.

No es tarea fácil porque es evidente que las diferencias de desarrollo en salud, entre cada país, pueden ser muy substanciales, incluso en poblaciones del mismo país, como ocurre en regiones del noreste de Brasil, norte de Argentina, comunidades rurales e indígenas de países andinos, comparadas con zonas urbanas de las 


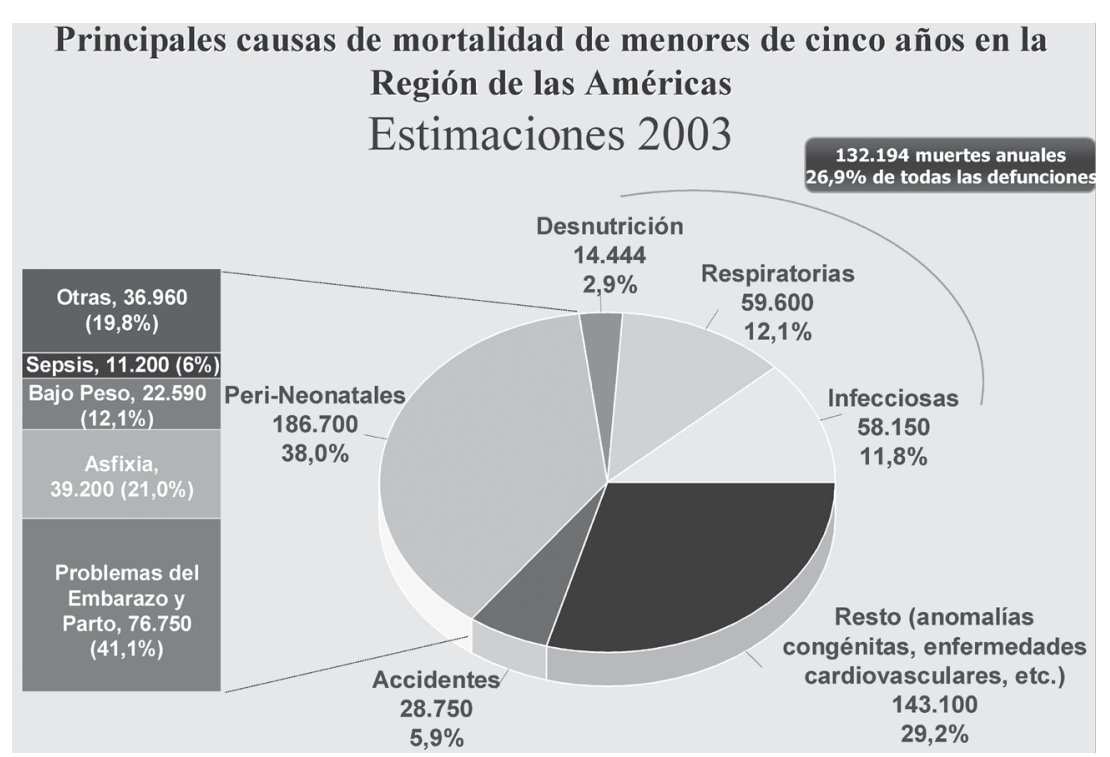

Figura 1. Fuente: Estimaciones de la Unidad de Salud del Niño y del Adolescente (FCH/CA), con base en datos de la Unidad de Análisis de la situación de Salud y Tendencias (AIS). Organización Panamericana de la Salud (OPS). 2004. mismas regiones. Para disponer de suficientes y apropiados datos será importante recabar de los antecedentes y resultados que organizaciones como OMS u OPS, han acumulado, así como revisar los datos epidemiológicos y bioestadísticos que en varios de estos países, son limitados, parciales, o definitivamente ausentes, de acuerdo a las publicaciones disponibles.

No obstante, las limitaciones y dificultades que entorpecen la tarea, es impostergable y urgente propiciar, definir y llevar a acción y efecto las prioridades programáticas y las intervenciones, que la región en general y cada país en particular, requieren y con dramática urgencia, especialmente las poblaciones más vulnerables y deprimidas.

La tarea es entonces, mejorar la salud ambiental (disminuyendo tasa de enfermedades infecciosas), aminorar la contaminación (que incrementa el número de enfermos respiratorios, asma, etc), evitar traumas y discapacidades originadas en accidentes y violencias, otorgando la apropiada disposición de agua potable a la población (previniendo diarreas, desnutrición, y enteroparasitosis). Protegiendo a las familias de la contaminación de los alimentos, del agua y de la tierra, en particular, entre las poblacio- nes más sensibles como los niños y embarazadas, respecto a químicos como los herbicidas, pesticidas, bifenilos, plomo, arsénico, o a radiaciones de distinta índole, cuyos efectos potenciales incluyen entre muchos otros daños, cáncer, trastornos genéticos, trastornos psicomotores, del comportamiento y de la inteligencia.

Respecto a los riesgos ambientales por la contaminación irresponsable, adquiere enorme importancia por las consecuencias que dramáticamente conocemos en el pasado reciente, y que adquiere en la actualidad, y especialmente para la seguridad sanitaria y la protección para una vida saludable que estamos otorgando a las generaciones futuras.

No hay duda que la calidad ambiental actual es diferente en casi todo a como se percibía y respiraba 40 a 50 años atrás. Desde el término de la segunda guerra mundial, la elaboración de químicos sintéticos no se ha detenido y en la actualidad existen más de 80 mil productos, de los cuales aproximadamente 3000 son potencialmente tóxicos y peligrosos para los niños; de estos químicos, se producen más de un millón de toneladas al año, que se distribuyen ampliamente en la atmósfera que respiramos o contaminan la tierra y los frutos con los que nos alimentamos. 
Ejemplo de las graves consecuencias se encuentran a diario, en la historia y en la memoria colectiva. Son ejemplos, entre otros muchos, los casos de niños australianos afectados por un brote de intoxicación plúmbica, los niños comprometidos en Antofagasta (Chile) por efecto del arsénico vertido al ambiente, los casos de Focomelia secundarios al uso de Thalidomida y los efectos del Dietilbestrol, en mujeres embarazadas, las miles de víctimas por consecuencia de las radiaciones en Hiroshima, Nagasaki, posteriormente en Chernobyl, y en días más cercanos, las graves contaminaciones químicas en nuestro país, como es el caso, de lo ocurrido en la zona de Valdivia, que afecta el ambiente y las personas, $\mathrm{y}$ ha sido motivo de reiterados llamados de resguardo y atención en diferentes publicaciones científicas, y por organizaciones que agrupan a profesionales e investigadores de la salud pública nacional.

Recientes comunicaciones dan cuenta del impacto que las diferentes amenazas ambientales afectan a los niños. Así por ejemplo en los países europeos la muerte de más de 22000 niños entre 0 y 4 años, es atribuida a la contaminación extra e intradomiciliaria, más de 13000 niños, entre 0 y 14 años mueren por causas originadas en deficientes condiciones sanitarias y aguas contaminadas, y los niños entre 0 y 4 años sufren la pérdida de alrededor de 480000 años de vida ajustados por discapacidad, ocasionados por la exposición al plomo y evaluado como retardo mental leve (definido con rango de Coeficiente intelectual entre 50 y 70 ).

En cuanto a las lesiones y traumas originados en accidentes y violencias, dichas referencias las describen como causa del $22,6 \%$ de todas las muertes entre los niños de 0 a 19 años, y del 19\% de pérdida de años de vida ajustados por discapacidad, en similar grupo de edad. Tales dramáticas cifras se repiten en otros lugares. En Estados Unidos de América, se refiere que el año 1999, 17940 niños menores de 19 años perdieron la vida por esta causa, cifra mayor que todas las otras causas de muerte combinadas. Se agrega además que la circunstancia origina 10 millones de consultas en las unidades de emergencia, y similar cifra en consulta en la atención primaria, y representa la principal causa de hospitalización en niños (aproximadamente 300000 ingresos por año).

En los países de América Latina las cifras son semejantes; en Chile por ejemplo, las lesiones derivadas de accidentes y violencias corresponde a la primera causa de muerte en los niños de 1 a 18 años, son la primera causa de hospitalización respecto a todas las otras causas en Hospital pediátrico en el área Metropolitana en Santiago. Ellos son además el principal origen de años de vida perdidos y muerte prematura, y representan el $5 \%$ de los egresos en Unidad de Cuidados Intensivos pediátricos estatales.

Aunque impresionan las cifras en cuanto a número de afectados, fallecidos y consultas por la circunstancia, el impacto económico es también enorme. Se publica que en EUA se gastan en cada año, billones de dólares en costos directos e indirectos. El año 1992 por ejemplo, se describe que el costo respecto de todas las lesiones en niños menores de 15 años, fue de 254 billones.

El costo de un programa de prevención en accidentes infantiles a nivel nacional que se estima, como se mencionó, logre una importante disminución de la morbilidad y una reducción de la mortalidad en alrededor de un $30 \%$, tendrá una relación costo beneficio enormemente favorable, aplicando medidas simples y prácticas, control y evaluación de actividades y resultados, y acotadas a la realidad epidemiológica de las distintas regiones del país. Dicha iniciativa implicará un compromiso transversal, que compromete a los poderes del estado en su conjunto, instituciones y organizaciones involucradas en la protección y educación del niño, y en especial, a la familia, informada y responsable inmediata del crecimiento integral del niño.

\section{Accidentes y amenazas en la infancia}

\section{Prevención y estrategias de intervención}

Transcribir las estrategias de intervención que incluyan todos las amenazas ambientales, supera ampliamente los márgenes de esta descripción, por lo que las páginas siguientes se corresponden y son referidas, a la prevención primaria de las lesiones, y sólo en aquellas originadas en traumas y accidentes. 
Como es reconocido, los daños provocados por accidentes deberán ser entendidos, como cualquier otra enfermedad, ya que posee un huésped (víctima o sujeto enfermo o lesionado), un agente que lo origina, interactuando con el ambiente, triada que define las condiciones o circunstancias, que hacen factible la lesión o injuria. Conceptualmente entonces será posible considerar intervenciones que podrán evitar o modificar el pre evento, el evento mismo y lo que sucederá posteriormente, y la acción que se realice, se hará sobre el agente causal, el huésped y la condición o circunstancia ambiental; el éxito de la intervención será mayor, cuando todas estas etapas son consideradas en su conjunto.

El enfoque actual del control de estas lesiones ha modificado el criterio de que los accidentes son esencialmente, eventos o circunstancias aleatorias ("mala suerte"), que no se pueden predecir y no son prevenibles, cambiando al concepto de que la mayoría de ellos, son evitables, de alguna manera predecibles y ocurren en situaciones o ambientes de riesgo, identificadas y potencialmente controlables.

Las intervenciones disponibles al respecto, se incluyen en tres grandes grupos:

- Promover cambios por medio de la educación, persuasión, o protección (en el caso de los niños menores).

- Impulsar las modificaciones en los ambientes de riesgo y estimular las modificaciones de las estructuras e ingeniería involucradas.

- Disponer las leyes que refuercen las medidas preventivas, disminuyendo los riesgos, sobre el huésped o vigilando que leyes o reglamentos sean cumplidos y/o los infractores sancionados, según corresponda.

Paradigma de lo descrito es la situación de un niño escolar, peatón que ha sido atropellado por un vehículo motorizado. En esta circunstancia la intervención incluirá la educación referida a enseñanza por medio del ejemplo que los padres le pueden otorgar al niño, respecto a la manera que se deberá comportar en la vía pública, educarlo respecto a leyes del tránsito, el uso de ropas reflectantes que lo protejan. En cuanto al ambiente, es probadamente útil la completa separación física entre vehículos y niños (vallas, pasos peatonales elevados, señales del tránsito, etc) y respecto al agente (vehículo) el control policial de la velocidad, la promoción de conductores responsables y respetuosos de las ordenanzas de tránsito, drásticas sanciones a los infractores, etc.

Acaecido el accidente y el trauma ulterior, la intervención incluirá la puesta en marcha de un adecuado sistema de rescate y transporte del lesionado, así como un eficaz manejo de la reanimación inicial en el lugar de accidente como en la unidad de emergencia (prevención secundaria).

Cabe recordar la trascendencia que ha tenido la obligatoriedad del uso del cinturón de seguridad para ocupantes de vehículos en Chile, y el impacto que ello ha implicado respecto al significativo aumento de su uso, y la consiguiente disminución de lesiones graves. Agregar el uso de sillas apropiadas en diseño y colocación, para niños pequeños en vehículos que los transportan, en EUA ha incidido en una disminución estimada de la letalidad en $69 \%$ entre los lactantes y de $47 \%$ en los niños mayores.

Los antecedentes de experiencias acumuladas, no obstante, permiten colegir que rara vez los cambios de conductas a través de la educación o persuasión son completamente exitosos, $\mathrm{y}$ las intervenciones deben necesariamente incluir todos los factores involucrados.

A mayor abundamiento, aquellos programas que han demostrado mayor eficacia, destacan enfatizar no sólo el riesgo o daño que pueden provocar determinadas conductas, sino que además las fortalezas y debilidades de los comportamientos alternativos, que incluyan por ejemplo incentivos en descuentos en el monto de los seguros para aquellos conductores no "accidentógenos", o que utilicen vehículos con aditamentos estructurales de seguridad, y multas o penas severas para los que conducen bajo los efectos de alcohol, drogas y/o contraviniendo gravemente las leyes del tránsito o utilizando vehículos en precarias condiciones.

A continuación se transcribe un análisis de las principales causas de accidentes graves en Chile, sus características epidemiológicas y una breve reseña de las medidas de prevención recomendadas en cada caso. 


\section{Lesiones derivadas: Su circunstancia y prevención}

En la tabla 2 se puede observar la distribución de niños fallecidos por edad y causas externas en Chile, en resultados proporcionados por el Instituto Nacional de Estadísticas, en el año 2000.

En el año 2000 en Chile murieron alrededor de 1000 niños por las denominadas causas externas. Tres tercios de las muertes fueron no intencionales. La principal causa de deceso fueron los originados en accidentes automotores, como peatones y ocupantes. La asfixia por inmersión fue la primera causa aislada de muerte. Esta, junto a las quemaduras afecta preferentemente a niños menores de 4 años. Como se observa además en la tabla 2, las lesiones intencionales representan una muy importante cifra de fallecidos, y comprometen especialmente a adolescentes mayores.

En esta tabla no se incluyeron los niños fallecidos cuyas causas se desconocen o se ignora si fueron intencionales o no.

\section{Accidentes en peatones}

Ninguna otra lesión provoca más pérdidas de vidas en los niños que los accidentes ocasionados por vehículos motorizados. Sin embargo, a diferencia de lo que ocurre en países desarrollados, la principal causa de accidente fatal es el provocado por atropello, actuando la víctima, el niño, como peatón.

Como es sabido, en nuestros países aún las condiciones de seguridad en que se desenvuelven los niños en calles y carreteras es muy precario, y está definitivamente confirmado que la disminución del número de niños que caminan solos (no acompañados de personas responsables) en la calle, aminora sustancialmente el número de lesionados. Ello explica la importante disminución (49\%) de víctimas fatales por

Tabla 2. Causas externas: Fallecidos agrupados de acuerdo a edad y circunstancia (INE. MINSAL. Chile, año 2000)

\begin{tabular}{|c|c|c|c|c|c|c|}
\hline & $\begin{array}{l}\text { Menor } 1 \\
\text { año }\end{array}$ & $\begin{array}{l}1 \text { a } 4 \\
\text { años }\end{array}$ & $\begin{array}{l}5 \text { a } 9 \\
\text { años }\end{array}$ & $\begin{array}{c}10 \text { a } 14 \\
\text { años }\end{array}$ & $\begin{array}{c}15 \text { a } 19 \\
\text { años }\end{array}$ & Total \\
\hline \multicolumn{7}{|l|}{ No intencionales } \\
\hline Asfixia inmersión & 10 & 64 & 4 & 26 & 61 & 165 \\
\hline Peatones & 0 & 35 & 32 & 24 & 44 & 135 \\
\hline Ocupantes vehículos & 3 & 16 & 21 & 17 & 57 & 112 \\
\hline Quemados & 8 & 30 & 15 & 9 & 18 & 80 \\
\hline Sofocación & 45 & 9 & 1 & 0 & 1 & 61 \\
\hline Intoxicación & 0 & 4 & 12 & 13 & 28 & 57 \\
\hline Caídas & 4 & 7 & 8 & 12 & 15 & 48 \\
\hline Ciclistas, motos & 0 & 0 & 0 & 1 & 10 & 11 \\
\hline Armas de fuego & 1 & 0 & 0 & 0 & 5 & 6 \\
\hline Procedimientos médicos & 2 & 0 & 1 & 3 & 2 & 8 \\
\hline Misceláneos & 5 & 15 & 20 & 0 & 0 & 40 \\
\hline \multicolumn{7}{|l|}{ Intencionales } \\
\hline Homicidios & 5 & 9 & 2 & 5 & 108 & 129 \\
\hline Suicidios & 0 & 0 & 0 & 14 & 114 & 128 \\
\hline Total & 83 & 189 & 116 & 124 & 462 & 968 \\
\hline
\end{tabular}


la causa en EUA; en Inglaterra se ha encontrado que la proporción de niños, escolares entre 7 y 8 años de edad, que caminan sin compañía hacia o desde la escuela, ha disminuido de un $80 \%$ que era en 1971 a sólo el $9 \%$ en 1990 , lo que resultó en una disminución proporcional del número de lesionados.

Otros factores de riesgo importantes en este tipo de accidentes, son en primer lugar la edad; los niños más pequeños, especialmente aquellos del sexo masculino, están expuestos a las amenazas del tráfico las que exceden sus habilidades cognitivas, de desarrollo, comportamiento, sus condiciones sensoriales y físicas. La circunstancia es agravada ya que a menudo los padres sobrestiman las capacidades de sus hijos en su actuar como peatones, en los lugares de juego, en la calle, y son dañados cerca de su hogar, en estacionamientos o pasajes, por vehículos que al retroceder inadvertidamente los atropellan. Con trágica frecuencia los conductores son parientes o vecinos de la víctima.

Los niños mayores, entre 5 y 9 años, son particularmente vulnerables a este tipo de accidente, representan el grupo de edad más afectado, en especial niños varones que en consideración a sus perfiles de comportamiento y conducta, su falta de experiencia y su peculiar atrevimiento, muestran un riesgo dos veces mayor que las mujeres, y esta proporción se triplica u cuadriplica, cuando se registran sólo los lesionados críticos o con daño letal. Los niños son impulsivos, carecen de la capacidad de evaluar la velocidad, relación espacial y la distancia del vehículo que enfrentan. Sus capacidades auditivas, visuales, la percepción de profundidad, y habilidad para analizar el riesgo, se integran gradualmente y no son completamente maduras al menos, hasta los 10 años.

Otro factor de riesgo relevante y de más difícil resolución, es la condición socio económica del niño. Es ampliamente reconocido que el riesgo es inversamente proporcional a la condición económica y al nivel educacional de los padres. El problema se deriva en particular por los riesgos ambientales a que están sometidos, la falta de una protección apropiada de sus cuidadores, y la educación ejemplar que los padres puedan impartir a los niños mayores. $\mathrm{Su}$ quehacer se desenvuelve en comunas densa- mente pobladas, con escaso control policial sobre los conductores, ausencia de protectores de ambientes o señalizaciones y en especial una carencia de lugares de esparcimiento, deportivos o de juegos, protegidos, seguros y disponibles. Como se mencionó el niño caminando solo o jugando en la calle es el primordial riesgo.

De acuerdo a la tabla citada, la mayor frecuencia de lesionados ocurre por la acción imprudente de niños entre 5 y 14 años, en circunstancia de cruzar la calle en mitad de la cuadra. Los niños que sufren traumas con resultado fatal son habitualmente varones dañados en situación de juego, o en viaje desde o hacia la escuela, ocurre en horas de la tarde, en calles y carreteras de alto tráfico y que sufren a consecuencia del impacto, graves daños cráneoencefálicos o cervicales. Esta descripción caracteriza el perfil clínico y epidemiológico del niño chileno fallecido por accidente peatonal.

El análisis de 2690 egresos hospitalizados en una unidad de Cuidados Intensivos en un período de 8 años, entre 1993 y 2002, demostró que el $8 \%$ del total correspondieron a lesiones traumáticas derivadas de accidentes, el promedio de edad fue de 7 años, y un 90\% eran peatones, con franco predominio de varones. Trece de estos niños fallecieron, entre ellos, 10 por una lesión cráneo encefálica.

\section{Intervención}

Las lesiones a peatones requieren de una intervención muy compleja que incluye esfuerzos coordinados en distintos niveles y estamentos, y un manejo multifactorial, de muy diferente índole. Incluyendo sólo las medidas de mayor trascendencia, estas se pueden resumir en las siguientes actividades:

- Programas de entrenamiento de habilidades de los niños en riesgo en circunstancias y tiempo real de tránsito.

- Educación a los padres y cuidadores para que éstos enseñen a los niños con el ejemplo, de cómo comportarse en la calle.

- Modificaciones en la legislación y ordenanzas del tránsito.

- Cambios estructurales en los ambientes identificados como peligrosos (lomos de toro, pasarelas peatonales, apropiadas señales e información de tránsito y tráfico) y avances 
en el diseño y seguridad de vehículos motorizados (alarmas al retroceder, espejos posteriores, barras anti vuelco, etc.)

Los cambios estructurales en el ambiente son intervenciones de alto costo monetario, sin embargo, para ser eficaces se requiere un cambio conductual en los usuarios. Por ejemplo los pasos peatonales separan efectivamente a los peatones del tráfico vehicular, sin embargo, observamos cotidianamente como adultos y niños no los usan y burlan esta regla, agregando para sí y otros, nuevos peligros y daños.

A mayor abundamiento la información disponible en la literatura y en medios electrónicos, respecto a prevención es numerosa e interesante, y aplicable, y probablemente compatible, con la idiosincrasia y recursos de la familia latinoamericana. La organización Safe Kids, en EUA, ha definido por ejemplo, las "Normas básicas de seguridad para niños peatones", $\mathrm{cu}-$ yas recomendaciones transcribimos:

"Cruzar la calle sólo en la esquina o en los cruces de peatones señalizados, obedeciendo todas las señales del tráfico.

Caminar por la acera; si no la hubiere, hacerlo por el lado izquierdo de la calle enfrentando el tráfico.

Caminar siempre al menos hasta los 10 años, acompañado de un adulto.

Cruce sólo frente al colegio y cuando el conductor o persona acompañante, se lo indique. No cruce o se detenga por detrás de los vehículos o donde el conductor no lo pueda ver.

Toma la mano de un adulto cuando cruce la calle. Mire hacia la izquierda, derecha $e$ izquierda nuevamente antes de cruzar, y continúe mirando hacia ambos lados, hasta alcanzar el otro lado.

Si camina cuando ya está oscuro, use ropa reflectante y de colores brillantes, para que los conductores lo puedan ver. Usar una linterna es buena idea.

Si una mascota (perro) o juguete (balón), se encuentra en medio de la calle, pide a un adulto que lo traiga de vuelta.

Juegue sólo en el patio o en lugares habilitados para ello, lejos de la calle o los estacionamientos."
Se infiere entonces que lo principal será promover la protección de los niños pequeños mediante la mejoría de las condiciones de riesgo ambiental y persistir en la educación en prevención para niños mayores y adultos, y que estos puedan a su vez enseñar, con el ejemplo, a los menores el comportamiento seguro en las calles.

\section{Ocupantes de vehículos motorizados}

En los países desarrollados las lesiones ocurridas a ocupantes de vehículos en colisión, es la principal causa de muerte, siendo la mayor parte de las víctimas adolescentes entre 15 y 19 años.

En Chile, el número de lesionados es sustancialmente menor, aunque las cifras de los últimos años muestran un progresivo incremento especialmente entre adolescentes y adultos jóvenes del sexo masculino.

\section{Intervención}

a. En lactantes, preescolares y niños menores de 8 años:

El uso de sillas y otros elementos de fijación al interior de vehículos motorizados, para niños menores de 5 años ha demostrado prevenir en más del $70 \%$ las muertes por la causa. En circunstancia de una colisión, el uso de cinturones o sillas de seguridad apropiados serán fundamentales para evitar los daños, o lesiones fatales, sin embargo, en muchas ocasiones estos equipos no son utilizados o son inadecuados. Es reconocido el peligro que se agrega al niño ocupante, que usa un cinturón de seguridad que pasa muy alto por sobre su pecho o cuello, o sobre su abdomen, lo que provocará serias lesiones en cuello, cara y en intestino o cráneo respectivamente. La recomendación actual es que los niños menores de 8 años, viajen en asientos suplementarios, con cinturones de falda y en bandolera. Ello evitará los riesgos que los afectan al utilizar cinturones de seguridad para adultos. Todos los niños menores de 12 años deben viajar en el asiento trasero.

Se describen y recomiendan distintos tipos de sillas o equipos de seguridad, de acuerdo a la edad o peso de los usuarios. Es así que en 
lactantes hasta $10 \mathrm{k}$, se usará una silla de lactante, ubicada en asiento posterior, mirando hacia atrás, con bandas tipo arnés a la altura de los hombros o debajo de ellos. En niños entre 10 y $18 \mathrm{k}$, la silla se ubicará en el asiento posterior mirando hacia adelante, con correas del arnés en o por encima de los hombros, y para los niños mayores con peso sobre los $18 \mathrm{k}$, se usarán los cinturones de seguridad de dos puntas, con asiento suplementario para ubicarlo, cuidando que se adosen firmemente sobre pelvis, la correa inferior y cruzando sobre pecho y hombro, la superior.

Utilizar los asientos posteriores es más seguro que los frontales (el riesgo de sufrir lesiones fatales disminuye en un $27 \%$ ), además se considera que las bolsas de aire frontales aumentan el riesgo de muerte para menores de 13 años, razón por lo cual siempre el lugar más seguro para los niños son los asientos posteriores. Todas las medidas de protección y control descritas, deben obviamente ser implementadas estrictamente en vehículos y conductores de transporte escolar; en nuestro país, desgraciadamente, se observa escaso rigor en el control y cumplimiento de las normas y ordenanzas al respecto.

En este contexto entonces, se puede afirmar fehacientemente que la educación en prevención y las medidas legislativas de intervención, dan excelentes resultados y disminuyen significativamente el número de víctimas originadas en este tipo de accidente y se constituye en la tarea y objetivo, por requerir de autoridades y comunidad organizada.

\section{b. En adolescentes:}

Como se ha descrito, las colisiones de vehículos motorizados es la causa más frecuente de muerte entre los adolescentes; en casuísticas recientes de EUA, se dice que el 50\% de los fallecidos son de este grupo de edad, y se agrega que un niño conductor de 16 años, tiene 7 veces más posibilidades de chocar que una persona de 25 años. El riesgo es aún mayor si el conductor es del sexo masculino.

Los principales factores de riesgo involucrados para esta edad y circunstancia, son los siguientes:
- No usar el cinturón de seguridad.

- El número de pasajeros viajando con un conductor adolescente: se estima que el riesgo de muerte aumenta en un 50\% cuando viaja con un pasajero e incrementa proporcionalmente al número de acompañantes, $\mathrm{y}$ el riesgo aumenta aún más, si estos son menores de 30 años.

- Conducir bajo los efectos del alcohol o drogas y durante las horas de la noche: Tal circunstancia aumenta el riesgo de colisiones a todas las edades, sin embargo, esta condición es particularmente importante en adolescentes. En Chile, la ley pena a los conductores con niveles de alcoholemia sobre $0,1 \mathrm{gr} \%$, no obstante las habilidades para conducir se ven afectadas con niveles aún bastante menores. Reconociendo esta situación muchos países han adoptado la estrategia de tolerancia cero para alcohol entre adolescentes. Esta medida ha dado como resultado una disminución sustancial de las víctimas fatales, entre este grupo de edad. Los conductores adolescentes tienen entre 5 a 10 veces más posibilidades de sufrir choques fatales en la noche, que durante el día. En estas circunstancia, la triada letal es la combinación de un adolescente, inexperto, que cree ser "inmortal", conduciendo en la noche, y bajo los efectos del alcohol.

Algunas diferentes estrategias se han organizado para intentar el control del problema, entre las más promisorias y aparentemente efectivas, se menciona el programa de capacitación para conductores jóvenes egresados de enseñanza media, cuyo propósito es mejorar las habilidades y experiencia en la conducción, previniendo su participación en situaciones de alto riesgo. El programa incluye licencia provisoria controlada y restricciones, como la conducción nocturna en días festivos o prefestivos, control en el número de pasajeros, uso obligatorio del cinturón de seguridad, tolerancia cero para alcohol, etc. La implementación y la aplicación controlada de tales iniciativas en nuestro país, permitirá reconocer sus efectos y eficacia, y en ese caso su necesaria y amplia difusión. 


\section{Asfixia por inmersión}

Los niños fallecidos en Chile por esta circunstancia aislada, supera en número absoluto a los decesos sufridos por peatones y representa entonces la primera causa de muerte por accidentes, demostrando su trascendencia e importancia como amenaza para nuestra población infantil. Algunas de sus principales características se transcriben a continuación:

- Ocasionan un alto número de casos fatales por evento.

- El pronóstico se relaciona directamente con las acciones que se realicen en los primeros diez minutos de ocurrido el accidente, es con esta consideración que la disminución de la morbilidad y la mortalidad se logrará preferentemente con la prevención primaria (evitar que el accidente suceda).

Los lugares de ocurrencia varían de acuerdo a la edad, entre los menores de 2 años el accidente se origina en el interior de la casa o en sus cercanías inmediatas como en la tina de baño o en baldes o recipientes de líquidos disponibles en los hogares. En los preescolares el ahogamiento sucede en piscinas o cursos de agua aledaños al hogar (acequias, canales).

Entre los adolescentes y niños mayores tal circunstancia ocurre en ríos, lagos, en el mar, durante actividades recreativas o deportivas, y con frecuencia bajo los efectos del alcohol o drogas, y afectando preferentemente a niños del sexo masculino.

\section{Intervención}

Las medidas de protección en los niños más pequeños para la prevención de estos accidentes en el hogar, requieren de la actitud responsable y advertida de adultos y cuidadores. Procurar mantener una vigilancia continua, omitiendo situaciones y acciones de riesgo en el ambiente doméstico, por ejemplo, evitar distracciones durante el baño, que por sólo un minuto pueden ser fatales, no mantener en casa receptáculos o cubos con líquidos en su interior, recordando que bastan pocos centímetros de agua acumulada, para que sufran ahogamiento.

En niños entre 1 y 9 años, el principal peligro proviene de piscinas y cursos de agua cercanos al domicilio, el accidente ocurre con frecuencia cuando estos juegan o caminan por los bordes. En consideración a esta circunstancia se requiere una constante y cercana supervisión por personas adultas responsables. La principal medida ambiental de prevención primaria (pasiva), es el uso de rejas o mallas que delimiten el perímetro de piscinas o canales, la recomendación es que estas posean una altura no menor de 1,5 metros, que su estructura no permita su escalamiento o traspaso por menores, y que cuente con una puerta segura. No se recomienda por inefectivas el uso de alarmas o cubiertas en la superficie del agua.

La recomendación actual es que la enseñanza de la natación sólo comience desde los 5 años de edad; esta práctica realizada en edades más tempranas acarrea riesgos comprobados, como intoxicación hídrica, infecciones, convulsiones, etc, y otorga una falsa y peligrosa sensación de seguridad para padres y cuidadores. Los niños de estas edades no tienen la suficiente habilidad psicomotora, carecen del suficiente autocontrol en situación crítica, ni poseen la experiencia para superar el imprevisto de caída al agua y el sofocamiento inicial. Esto no excluye obviamente toda la apropiada enseñanza que pueden proporcionar expertos, a los niños respecto a seguridad y comportamientos en el agua.

Otros factores destacados relativo al riesgo entre los niños mayores y adolescentes, es la habitual práctica de nadar o efectuar buceo en cursos de agua inseguros, como ríos o en el mar, y su participación en actividades recreativas o deportivas acuáticas sin los implementos de seguridad adecuados, y practicarlas bajo los efectos del uso de alcohol o drogas (que afecta a cerca del $50 \%$ de las víctimas adolescentes).

Es importante recomendar el uso de chaquetas salvavidas individuales, apropiadas a la edad y peso de los niños, en todas las circunstancias, juegos o deportes acuáticos, y durante actividades recreativas cercanas a ríos, mares o lagos. No se aconseja utilizar otros sistemas de flotación alternativos, de muy profusa difusión comercial y que otorgan inadecuada protección y escasa seguridad en el agua (juguetes plásticos inflados o "alitas", por ejemplo).

Es útil también enseñarles el riesgo que 
implica nadar solos o sin la supervisión de adultos, y en los adolescentes representarles el peligro que significa el uso de alcohol o drogas durante estas actividades. La prohibición del expendio de bebidas alcohólicas en playas y balnearios es una medida de prevención primaria recomendable.

Entre otras modalidades de prevención secundaria, es importante la capacitación de la comunidad en reanimación cardiopulmonar básica, para niños y adultos, habilidades que ocupan un lugar destacado por su trascendencia, procurando en niños y adultos, el apropiado aprendizaje de las destrezas necesarias y exigibles, capacitándolos para actuar en situaciones de urgencia crítica y rescate, maniobras cuyo efecto y consecuencia, se relacionan directamente con el pronóstico del evento.

\section{Quemaduras}

En Chile, las quemaduras son la cuarta causa de muerte accidental entre los niños mayores de 1 año, son además fuente de gran sufrimiento para los afectados, provocando un enorme impacto personal, social y económico para las víctimas y su familia, así como cuantiosos costos en su manejo y rehabilitación, dejando perdurables cicatrices que acompañarán al niño y le afectarán radicalmente en su retorno a una vida emocional normal.

Las quemaduras afectan más frecuentemente a los lactantes mayores y preescolares, de ambos sexos, ocurren de preferencia en el hogar, durante los meses de invierno y en regiones de clima frío, afectando a familias de situación socioeconómica precaria, en condiciones de hacinamiento, con escasas medidas de protección.

Se originan principalmente por escaldaduras, contacto con estufas (braseros), u otros objetos calientes, por manipulación de equipos eléctri$\cos \mathrm{y}$ en incendios, causados por cigarrillos encendidos, juegos con fósforos, fallas en sistema eléctrico, etc.

Las lesiones por líquidos calientes son más frecuentes en niños menores de 4 años, sufren quemaduras más profundas, afecta una mayor proporción de superficie corporal que el adulto, en contacto con similar cantidad de energía térmica.

La mayoría de los decesos por causa de incendio, suceden en el lugar del accidente y se originan por inhalación de humo y en ocasiones cuando los niños pequeños son mantenidos en habitaciones cerradas, donde tienen escasas posibilidades de escapar en una situación de incendio.

Se recordará la trágica circunstancia de muerte de decenas de niños discapacitados que murieron en horas de la noche, atrapados en habitaciones de su Hogar Niño y Patria, en un recinto con las puertas cerradas con llave, ventanas con reja metálica, y el cuidador que se percató del fuego cuando nada podía hacer por ayudarlos. Situación y consecuencias semejantes ocurrieron hace algunos años también en el Pequeño Cottolengo.

En EUA se estima que cada año sufren quemaduras 1200000 personas, 60000 de éstas son hospitalizadas y aproximadamente mueren 6 000, son víctimas especialmente aquellos que se encuentran en los extremos de la edad, son niños pequeños y ancianos los que sucumben en mayor proporción. Hechos y cifras que dan cuenta de la magnitud e importancia de este accidente como problema relevante de salud pública y representan el desafío más crítico, demandante y complejo que enfrenta el personal hospitalario, entre toda la amplia gama de lesiones traumáticas.

\section{Intervención}

Las medidas de prevención primaria obvias al respecto incluyen, la protección de los niños más pequeños por parte de padre y cuidadores. Resolver la exposición al riesgo, tanto en ambientes como en acciones peligrosas, cuya eficacia limita con las condiciones socioeconómicas y culturales actuales de muchas familias chilenas, que requerirían cambios estructurales en las viviendas y modificaciones en las conductas y comportamientos, y de padres más advertidos y responsables; tales medidas son sin embargo, caras y a largo plazo las primeras, y representan un esfuerzo educacional sistemático y permanente en las últimas.

En este contexto es recomendable utilizar y exigir el uso en los hogares, de un sistema de 
desconexión denominado Interruptor térmico magnético (automático), en la red eléctrica domiciliaria, que impide el sobrecalentamiento de los conductores y el riesgo de incendios. No obstante el elemento de mayor importancia para evitar la electrocución o las quemaduras de este origen es el interruptor diferencial, que instantáneamente bloquea el paso de la corriente eléctrica cuando se produce el contacto, elemento de fácil instalación y de costo muy bajo, en consideración a los graves daños que previene. De la misma manera se aconseja procurar que cocinas y hervidores eléctricos, se encuentren fuera del alcance de los niños, y que su diseño y manipulación se asegure evitar el contacto o derrame de líquidos calientes sobre ellos.

Se reconoce que el riesgo de muerte en incendios puede disminuirse en la mitad de las víctimas, cuando se instala en el hogar, y especialmente en centros, hogares, internados, escuelas y salas cunas donde viven, pernoctan o duermen los menores, (iy también en hogares de los ancianos y los discapacitados de cualquier edad!), adecuadas alarmas de humo con baterías de Litio, de duración prolongada, e independientes de la red eléctrica instalada, permite asegurar un aviso temprano y oportuno en caso de ignición o incendio.

En este mismo sentido los padres deben aprender que los niños pequeños requieren una supervisión muy cercana y permanente, nunca los niños menores se deben encontrar solos en el hogar, enseñar a los mayores de 3 años como comportarse en caso de fuego en la casa, advertirles que la más segura manera de escapar, es gateando sobre la superficie del piso, cuando la pieza está llena de humo o gases tóxicos; se reconoce que el aire más limpio se encuentra precisamente entre 30 a $60 \mathrm{~cm}$ del suelo; discutir y practicar con ellos un plan de escape en el hogar (también en colegios y escuelas). Para aquellos que viven en departamentos y edificios de altura, enseñar que los ascensores no se deben utilizar en caso de incendio porque estos pueden detenerse justamente en el lugar del fuego, y es perentorio usar sólo las escaleras en la emergencia.

Se mostrará a los niños que cuando sus ropas se están quemando, deben detenerse (no correr), lanzarse al suelo y rodar, para apagar las llamas, y los primeros auxilios consistirán en aplicar en forma inmediata y profusamente agua sobre las superficies cutáneas afectadas.

Respecto a este accidente como en otros, el pediatra debe considerar transmitir consejos y recomendaciones a los padres y cuidadores, durante la atención primaria, que les permita acrecentar su educación en prevención, por ejemplo, estimular a adultos y adolescentes para no fumar o no hacerlo en el hogar, aprender el uso apropiado de los equipos extintores y aportarles información tanto oral como escrita, relativa a capacitación en la atención inicial, que en ocasiones es muy importante para disminuir morbilidad y mejorar el pronóstico.

Considerar con padres, profesores y autoridades, la importancia que supone la educación al respecto y la inclusión de contenidos de información y prevención en el currículo escolar.

La Rama de Accidentes, Envenenamientos y Violencia de la Sociedad Chilena de Pediatría, en conjunto con el Ministerio de Salud y la empresa privada, ha editado y promueve el diseño de folletos y afiches para su distribución y difusión en la atención primaria. Este material estará disponible para los profesionales de la salud y educadores, con el propósito de su extensión y difusión en la comunidad.

\section{Caídas y lesiones en actividades recreativas o deportivas}

Las caídas son las circunstancias más frecuentes como origen de lesiones traumáticas en la niñez, usualmente provocan daños menores y representan más de la mitad del total de consultas en los Servicios de Urgencia de nuestro Sistema de Salud Estatal.

Ocurren cuando el lactante comienza sus desplazamientos, progresa en sus capacidades motoras, e intenta ponerse de pie y da sus primeros pasos, o se encuentra jugando. La mayor parte de las lesiones son leves, sin embargo, las denominadas caídas de alturas máximas, pueden ser graves y ocasionalmente mortales.

Estos accidentes suceden preferentemente en el hogar, afectando a los lactantes mayores 
y preescolares, con un ligero predominio entre las mujeres $(57,3 \%)$. Posteriormente, ya entre niños mayores de 5 años los daños ocurren fuera de la casa, durante actividades de esparcimiento o realizando actividades deportivas o físicas, donde predominan los lesionados del sexo masculino.

Las consecuencias más frecuentes son lesiones menores, como heridas o contusiones que hacen más del 50\% del total de los consultantes en los servicios de urgencia, no obstante un grupo importante de ellos sufre traumas graves, como fracturas, cuyo número alcanza en un año a más de 3600 afectados (tasa de 1200 por 100000 de población entre 1 y 14 años, en Área de Salud Metropolitana Sur), muchos de los cuales requieren hospitalización, cirugía y tratamientos ortopédicos complejos.

Se suman a estos, los niños que sufren lesiones cráneo encefálicas, que representan aproximadamente la causa del $80 \%$ de las muertes por trauma, donde predominan escolares y adolescentes del sexo masculino, y cuyo orígenes fueron en orden de mayor a menor frecuencia, los atropellos a peatones, los daños en ocupantes de vehículos de 2 y 4 ruedas, las caídas de altura, y otros misceláneos, donde se incluyen agresiones y violencias por terceros o maltrato infantil.

Se describe que entre el 25 al $50 \%$ de las lesiones cráneo encefálicas en niños menores de 2 años tiene como origen el maltrato grave. Es importante destacar aquí que con cierta frecuencia padres y cuidadores, aducen las caídas como circunstancia que relatan, para esconder lesiones falsamente accidentales, y que corresponden realmente a abusos o negligencias.

\section{Intervención}

Las estrategias en la prevención de las caídas poseen diferentes modalidades de acuerdo a la edad de los afectados; así entre los niños menores de 4 años será necesario extremar las medidas de protección, controlando y vigilando sus desplazamientos, otorgándoles espacios seguros y libres de riesgos ambientales como corrales para los más pequeños, uso de barandas en escaleras, rejas en ventanas, y preparándoles lugares de juegos apropiados, con superficies de piso de arena $\mathrm{u}$ otros materiales que amortigüen las consecuencias de las caídas.

No se aconseja el uso del andador, artefacto muy popular entre las madres por la comodidad que representa ubicar allí, especialmente a los niños más inquietos, pero muy peligrosos para la integridad del menor, pues les facilita su desplazamiento, lejos de la supervisión de los mayores, y cerca de los lugares más riesgosos de la casa, como cocina, balcones, escaleras, etc.

En los preescolares que ya tienen más capacidad e independencia motora, con amplio espacio exterior para sus actividades lúdicas, adquiere gran trascendencia la tarea de padres y cuidadoras en el control y vigilancia cercana, evitando lugares de riesgo comprobado como estacionamientos de vehículos, juegos infantiles diseñados en altura, escalas o toboganes con materiales estructuralmente inapropiados.

En nuestro país el número de niños que practica ciclismo u otra actividad deportiva en vehículos con ruedas como patines, skates, etc., es progresivamente más numeroso y se ha extendido a poblaciones de diferentes estratos; desgraciadamente el uso del casco protector no se practica en similar proporción y las lesiones por caídas, impactos sobre cara y cráneo siguen ocurriendo.

Se reconoce que utilizar el casco, disminuye el riesgo de lesión craneoencefálica en alrededor del $80 \%$ de los ciclistas y sobre la cara en un $65 \%$, no obstante lo cual, rara vez vemos ciclistas usándolo, y ello sucede probablemente por factores como incomodidad, modas o ignorancia.

Aunque la educación respecto a la autoprotección y seguridad personal es importante, parece ser más efectiva la legislación que controle y sancione tales conductas, y entregue normativas respecto a la venta de bicicletas y otros vehículos deportivos que necesariamente incluirá la adquisición del adecuado casco. En los países desarrollados el estado bonifica parcialmente el precio de este, al considerar la positiva relación costo beneficio que implica su utilización; se estima que por cada dólar que se gasta en un casco de ciclista, la sociedad ahorra 30 en costos médicos y otros gastos. El valor de un casco en Chile no supera los \$20000. 
En similar contexto, los padres tienen una responsabilidad principal, pues se describe que la mayoría de los niños usarán el casco si ven a sus padres usarlos y ésta enseñanza es más efectiva si se inculca desde la más corta edad de los niños y al iniciar el aprendizaje; son valiosos también sus consejos sobre los lugares apropiados y seguros para esta práctica, la no conducción en horas de la noche, en calles con alto tráfico vehicular y con tránsito en dos sentidos, y la necesidad que estos conductores aprendan y respeten las leyes y normas de tránsito.

Los mismos conceptos estratégicos en prevención se deben aplicar en otras actividades deportivas especialmente populares entre los adolescentes, como motociclismo, motos de agua, playa o nieve, ski y equitación. En este escenario las caídas ocasionan con alarmante frecuencia serios traumas cráneo encefálico y cervical, donde también el casco representará un rol significativo en la prevención o disminución de los daños.

\section{Intoxicaciones}

Esta circunstancia provoca aproximadamente entre el 4 y el $7 \%$ de las consultas en los Servicios de Urgencia Infantiles. En el Hospital E. González Cortés, la cifra alcanza a alrededor del 4,5\% y representa 1300 consultas anuales por la causa. De acuerdo a las informaciones comunicadas por CITUC, (Centro de Información Toxicológica, Universidad Católica de Chile), en Santiago, las consultas anuales por la causa alcanzan entre 38000 y 49000 , estas cifras acreditan la importancia del problema como fuente de morbilidad.

Un pequeño número de los niños afectados necesitará hospitalización (7\%), con frecuencia su admisión se efectuará en las unidades de paciente crítico, por su condición de gravedad o por requerir una estricta vigilancia de sus signos vitales.

Los antecedentes respecto a decesos por esta causa en Chile muestran un número importante de casos (57 durante el año 2000), de misceláneos orígenes, acumulados con mayor frecuencia entre los niños escolares y especial- mente adolescentes, entre ellos los que cometen suicidio, por ingestión de medicamentos, preferentemente del sexo femenino.

Interpretar estos datos es complejo, probablemente muchos de estos niños han sufrido sobredosis de drogas ilegales o alcohol, o son efectivamente suicidios mal clasificados, a ellos se deben sumar niños víctimas de tóxicos ambientales, como plaguicidas, órgano fosforados por ejemplo; intoxicados por monóxido de carbono; por ingestión de medicamentos como antidepresivos tricíclicos o por reacciones sistémicas anafilácticas (picadura de insectos), o mordedura de araña por Loxoceles Laeta. Al respecto Schenone y colaboradores, recientemente han comunicado un estudio en 56 pacientes afectados, (entre los años 1955 y 2000), de los cuales el $68 \%$ sufrió compromiso cutáneo y $32 \%$ cutáneo visceral con una letalidad, entre estos últimos del 22,2\%.

\section{Intervención}

Las medidas recomendadas para la prevención primaria incluye la educación y difusión entre padres y cuidadores de las situaciones de riesgo y los ambientes peligrosos en el hogar, limitando el acceso de niños menores a medicamentos, por ejemplo promoviendo el diseño y distribución de tapas de seguridad en los envases, envasando substancias químicas, insecticidas y plaguicidas en receptáculos apropiados y lejos del alcance de los niños, enseñándoles como protegerse de amenazas ambientales como insectos, animales y plantas venenosas.

Reconociendo además las fuentes de producción de tóxicos en el ambiente, como monóxido de carbono en el domicilio, originados por medios inseguros de calefacción (braseros, estufas), prohibiendo la construcción y habilitación de baños con calefón en su interior, que son fuente de peligrosa y con cierta frecuencia fatal emanación de gases tóxicos; se han descrito casos similares originados por la inhalación de gases producidos por motores de vehículos.

En cuanto a los niños mayores será importante la implementación de programas que promuevan su educación, comenzando en la etapa preescolar, con contenidos respecto al peligro sobre drogas peligrosas, marihuana, cocaína, alcohol y tabaco; muchos adolescentes en Chi- 
le, mueren o son víctimas de serias morbilidades cuando sufren accidentes o violencias, al encontrarse bajo los efectos de tales substancias.

Se agrega la importancia de informar a los futuros padres y adultos educadores, de los riesgos y la gravedad que representa para los fetos y recién nacidos que su madre use drogas adictivas, antes, durante el embarazo, después del parto, y de la misma manera como les daña el consumo de las denominadas drogas lícitas (alcohol, tabaco).

En un contexto más global es preciso destacar la trascendencia que para los niños y su familia, poseen todos los esfuerzos educativos e iniciativas multidisciplinarias y sectoriales que se promuevan para mantener un ambiente libre de contaminación y de tóxicos peligrosos, para el entorno.

Muy difundidos han sido los dramáticos casos ocurridos en la ciudad de Antofagasta, respecto a la intoxicación por arsénico en la comunidad, y la situación emergente que se presenta con la diseminación del plomo en el medio ambiente, elemento considerado entre los más dañinos para la salud infantil, especialmente por afectar el desarrollo neurológico y conductual de los afectados. Testimonio de la silenciosa y peligrosa amenaza por este tóxico, son los trabajos efectuados por el CEDIUC (Centro de Diagnóstico Universidad Católica) que mostró que un $17 \%$ de los niños controlados mostraban un nivel superior a $10 \mathrm{mg} \%$ en su sangre, relacionando el riesgo con la cercanía de la fuente (las viviendas con calles de alto tráfico de vehículos contaminantes) con el domicilio de los afectados. También en Arica el año 2000 se realizó una investigación con resultados semejantes, encontrándose 120 niños con cifras en niveles tóxicos, de un total de 3.240 menores de 15 años a todos los cuales se les realizó la determinación de plomo sanguíneo.

Estos resultados hacen perentorio considerar las necesarias medidas de control en las fuentes de emisión, o evitando elementos que lo contienen o lo liberen al medio ambiente, entre estas, el contenido en la bencina de los motores de combustión, que se expele a la atmósfera, o el que se encuentra en baterías o en elementos metálicos y pinturas antiguas. Es importante recordar que el plomo no sufre alteraciones en contacto con la naturaleza y sus efectos nocivos persisten contaminando aguas y tierras, vegetales y animales, por largo tiempo.

Relativo a prevención, ha sido muy notable la gestión sanitaria que ha desarrollado el CITUC, respecto al qué hacer en caso de emergencias, promoviendo la educación en la comunidad, así como el expedito y documentado respaldo técnico para servicios de urgencia y profesionales de la salud, permitiendo el acceso oportuno y consistente a la información y manejo de las intoxicaciones, durante las 24 horas del día.

\section{Conclusiones}

Se han descrito brevemente los accidentes más frecuentes y los más notables, que por su gravedad afectan al niño chileno, se ha hecho énfasis en la prevención de los más importantes, y se han enumerado las estrategias de intervención propuestas.

No obstante hay que admitir que la supresión completa de los accidentes infantiles no es posible; sin embargo, pertinente es plantear, de acuerdo con las organizaciones de salud internacionales, algunas recomendaciones fundamentales, cuando se intenta abordar el problema de una manera global y sistematizada, y que a continuación se transcriben:

- Abordar el tema desde un planteamiento programático multidisciplinario e intersectorial. Los accidentes en su proceso causal son multifactoriales.

- Promover en la población un cambio radical en las actitudes y conductas hacia el fenómeno del accidente y sus consecuencias. Revisar y actualizar normas y legislación apropiada a estos efectos.

- Contar con un soporte informativo básico, que incluya datos respecto a circunstancias, naturaleza y gravedad, asistencia recibida y estimación de los daños. Estos antecedentes se utilizarán para definir prioridades en prevención y evaluar los resultados de las medidas adoptadas.

- Conocer la epidemiología y los factores humanos y ambientales involucrados, será el fundamento de las acciones y estrategias, en educación participativa y adherida al cu- 
rrículo escolar, general y permanente. Asistencia y control de las modificaciones del ambiente que correspondan.

- Promover una asistencia médica adecuada, desde la capacitación en los primeros auxilios por personas comunes. Procurar el desarrollo de una red nacional y regional de rescate, transporte profesional, y la apropiada habilitación del recurso humano y físico en unidades de emergencia, generales de trauma y las neuro quirúrgicas, correspondientes con la realidad epidemiológica de cada región.

- Incorporar el entrenamiento de la población para las actuaciones de urgencia y catástrofes.

Creemos que los pediatras poseen una singular responsabilidad para justificar en propiedad su apelativo de "defensores del niño". Es nuestra tarea entonces, crear y desarrollar en cada comunidad, programas de educación y prevención, respecto a riesgos y daños en accidentes, así como capacitarse y aprender, para luego enseñar, métodos y destrezas para que el manejo de los niños lesionados sea eficaz y oportuno.

\section{Referencias}

1.- Instituto Nacional de Estadísticas. Ministerio de Salud. Servicio de Registro Civil e Identificación. Anuario de Estadísticas Vitales 2000.

2.- Comisión Nacional de Seguridad en el Tránsito. Secretaría Ejecutiva: Fichas para la Acción 1 a 54. $\mathrm{http} / /$ :www.conaset.cl

3.- Mena A, Romero P: Accidentes infantiles en Chile. UNICEF. Sociedad Chilena de Pediatría. 1995.

4.- Johnston B, Rivara F: Injury Control: New challenges. Pediatr Rev 2003; 24: 111-7.

5.- Brent R: Addressing Environmentally Caused Human Birth Defects. Pediatr Rev 2001; 22: 153-65.

6.- Rivara F, Grossman D: Prevention of traumatic death to children in the United States. Pediatrics 1996; 97: 791-7.

7.- $Y u$ S: The life-course approach to health. AJPH 2006; 96: 768 .

8.- Thernitchin A: Ambiente y salud. La contribución médica. Cuad Med Soc (Chile) 2005; 45: 75-6.

9.- París E: Pediatría Ambiental. Pediatría al Día 2005; 21: 3 .

10.- Valent F, Little D, Bertollini R, Nemer LE, Barbone F, Tamburlini G: Burden of disease attributable to selected enviromental factors and injuries among Europe's children and adolescents. Lancet 2004; 363: 2032-9.

11.- Goldman LR: The clinical presentation of environmental health problems and the role of the pediatric provider in Childrens environmental health. What do I do when I see children who might have an environmentally related illness? Pediatr Clin North Am 2001; 48: 1085-98.

12.- Blanco E: Tabaco, salud y sociedad. Cuad Med Soc (Chile) 2005; 45: 775-86.

13.- Hollstein D, Vega J, Carvajal J: Desigualdades sociales y salud. Nivel socioeconómico y mortalidad infantil, 1988-1995. Rev Med Chile 1998; 126: 333-340.

14.- Cáceres D, Adonis $M$, Retamal C, et al: Contaminación intradomiciliaria en un sector de extrema pobreza de la comuna de La Pintana. Rev Med Chile 2001; 129: $33-42$.

15.- Edwards P, Roberts I, Green J, Lutchum S: Death from injuries in children and employments status in family: analisis of trends in class especific death rates. BMJ 2006; 333: 119.

16.- Prado V, Solari V, Álvarez IM, et al: Situación epidemiológica de las enfermedades transmitidas por alimentos en Santiago de Chile. Período 1999-2000. Rev Med Chil 2002; 130: 495-501.

17.- Dowd D, Keenan H, Bratton S: Epidemiology and prevention of childhood injuries. Crit Care Med 2002; 30: 385-90.

18.- Romero P: Prevención de accidentes en la infancia. Pediatría al día 2005; 21: 36-45.

19.- Cummings $P$, Rivara $F$, Olson $C$, et al: Changes in traffic crash mortality rates attributed to use alcohol, or lack of a seat belt, air bag, motorcycle helmet or bicycle helmet, United States, 1982-2001. Injury Prevention 2006; 12: 148-54.

20.- Quinones-Hinojosa A, Jun P, Manley G, Knudson M, Gupta N: Airbag Deployment and Improperly Restrained Children: A Lethal Combination. J Trauma 2005: 59: 729-33.

21.- American Academy of Pediatrics. Committee on Sports Medicine and Fitness and Committee on Injury and Poison Prevention. Pediatrics 2000; 105: 868-9.

22.- Meyer R, Theodorou A, Berg R: Childhood Drowning. Pediatr Rev 2006; 27: 163-9.

23.- American Academy of Pediatrics. Committee on Injury and Poison Prevention Reducing the number of deaths and injuries from residential fires. Pediatrics 2000; 105: $1355-7$.

24.- Ramzy P, Barret J, Herndon D: Thermal Injury. Crit Care Clin 1999; 15: 333-52.

25.- París E, Ríos JC: Intoxicaciones. Epidemiología, clínica y tratamiento. Ed. Universidad Católica de Chile. 2000.

26.- Laraque D, Trasande L: Lead poisoning: Successes and 21 st century challenges. Pediatr Rev 2005; 26: 435-43.

27.- Duhaime $A C$ : Non accidental head injury in infants the "shaken baby syndrome". N Engl J Med 1998; 338: 1822-9.

28.- Schenone H, Rubio S, Saavedra T, Rojas A: Loxocelismo en Pediatría. Región Metropolitana. Rev Chil Pediatr 2001; 72: 100-9. 